\title{
Aspirin enhances the cytotoxic activity of bortezomib against myeloma cells via suppression of Bcl-2, survivin and phosphorylation of AKT
}

\author{
JIANG-HUA DING $^{1}$, LI-YA YUAN ${ }^{2}$ and GUO-AN CHEN ${ }^{3}$ \\ ${ }^{1}$ Hematology and Oncology Department, The No. 171st Hospital of PLA, Jiujiang, Jiangxi 332000; \\ ${ }^{2}$ Hematology Department, Jiangxi Academy of Medical Science; ${ }^{3}$ Hematology Department, \\ The 1st Affiliated Hospital of Nanchang University, Nanchang, Jiangxi 330006, P.R. China
}

Received July 12, 2015; Accepted November 1, 2016

DOI: $10.3892 / \mathrm{ol} .2016 .5472$

\begin{abstract}
In our previous study, it was found that aspirin (ASA) exerted antimyeloma actions in vivo and in vitro. The resistance to bortezomib (BTZ) in multiple myeloma $(\mathrm{MM})$ is partly due to AKT activation and the upregulation of survivin induced by BTZ, which are the targets of ASA in gastric and ovarian cancer, respectively. Thus, the present study investigated the interaction between ASA and BTZ in $\mathrm{MM}$ and further clarified the underlying mechanisms. MM1.S and RPMI-8226 cell lines harboring the N- and K-Ras mutations, respectively, were treated with $2.5 \mathrm{mM}$ ASA, $10 \mathrm{nM}$ $\mathrm{BTZ}$ and ASA+BTZ for different durations. The proliferation and apoptosis of the cells were determined, and the underlying mechanisms governing the interaction of ASA and BTZ were examined in the MM cells. Treatment with ASA+BTZ caused higher rates of proliferative inhibition and apoptosis in the MM1.S and RPMI-8226 cells in time-dependent manner, compared with either agent alone. A drug interaction assay revealed the additive effect of ASA and BTZ on the myeloma cells. ASA alone inhibited the levels of phosphorylated AKT (p-AKT) and survivin, whereas BTZ alone augmented the levels of p-AKT and survivin. Of note, ASA markedly decreased the upregulation of $\mathrm{p}-\mathrm{AKT}$ and survivin induced by BTZ. Treatment with ASA+BTZ significantly suppressed the level of Bcl-2, compared with either agent alone. ASA may potentiate the antimyeloma activity of BTZ against myeloma cells via suppression of AKT phosphorylation, survivin and $\mathrm{Bcl}-2$, indicating the potential of ASA+BTZ in treating MM, particularly for cases of BTZ-refractory/relapsed MM.
\end{abstract}

Correspondence to: Dr Guo-An Chen, Hematology Department, The 1st Affiliated Hospital of Nanchang University, 17 Yongwai Zheng Street, Donghu, Nanchang, Jiangxi 330006, P.R. China E-mail: gachen923@hotmail.com

Key words: aspirin, bortezomib, multiple myeloma, survivin, B cell lymphoma-2, AKT phosphorylation

\section{Introduction}

Multiple myeloma (MM) is a malignant neoplasia of plasma cells, which accounts for $\sim 10 \%$ of all types of hematological cancer. According to the latest statistics, the annual incidence of MM was reported to be 20,450 cases and the mortality rate for MM was 11,090 cases/year in the USA in 2013 (1). In addition, MM has evolved as one of the major diseases affecting the aging population in China (2). At present, MM is the second most prevalent hematological malignancy following non-Hodgkin's lymphoma (2). Even with the introduction of immunmodulators, including thalidomide and lenalidomide, and proteasome inhibitors, including bortezomib (BTZ), over the last decade, the 5-year survival rate of patients in the USA with MM is only $42 \%$ (1). In addition, almost all patients with MM undergo relapse and refractory disease, predominantly due to drug resistance (3). Until now, MM remains an incurable disorder among hematological malignancies.

Patients with MM are at enhanced risk of thromboembolism due to disease-specific and treatment-specific risk factors, particularly when treated with thalidomide and lenalidomide $(4,5)$. Thus, the prevention of thrombosis has become important in cases of MM. At present, no significant differences have been observed among the use of aspirin (ASA), low-dose warfarin and low-molecular weight heparin in thromboprophylaxis (6). ASA may be the optimum candidate to prevent thromboembolism in patients with MM due to its merits in method of administration, safety and cost without the requirement for regular coagulation monitoring (7). ASA has been widely used for thromboprophylaxis in patients with MM, particularly in cases treated with thalidomide or lenalidomide combinations.

An increasing number of studies have demonstrated that ASA possesses antineoplastic actions against a wide range of solid tumor types, including esophageal, breast, lung and gastric cancer, and colon cancer in particular (8-12). Currently, the underlying anticancer mechanisms of ASA have been ascribed to the inhibition of nuclear factor (NF)-kB, and the induction of apoptosis by caspase activation, interruption of extracellular signal-regulated kinase and epidermal growth factor receptor in various types of cancer (13-17). Our previous study found 
that ASA exerted antiproliferative and pro-apoptotic activities in vivo and in vitro via the upregulation of B cell lymphoma-2 (Bcl-2)-associated $\mathrm{X}$ protein (Bax), and the suppression of Bcl-2 and vascular endothelial growth factor (18).

The antimyeloma effect of BTZ, the first proteasome inhibitor to be approved by the Food and Drug Administration of USA in 2003, is well-recognized. At present, BTZ has become the foundation of first- and second-line treatment in MM. The major mechanisms underlying the action of BTZ in MM include inhibition of the activation of NF-kB, upregulation of pro-apoptotic Noxa and the subsequent downregulation of anti-apoptotic Bcl-2 (19). However, the increasing administration of BTZ is associated with the development of drug resistance (20). There have been a number of explanations for the insensitivity to BTZ, including proteasome subunit $\beta 5$ mutation, insulin growth factor-1 and the differentiation status of myeloma cells (21-23). The activation of AKT and upregulation of survivin induced by BTZ itself are the primary factors involved in the development of resistance to BTZ in MM (24). Coincidentally, survivin, Bcl-2 and AKT have been confirmed to be the targets of ASA in gastric, cervical and ovarian cancer, respectively $(13,25,26)$.

In relation to the findings described above, the present study hypothesized that ASA in combination with BTZ may produce additive or synergistic effects in the treatment of MM. The aim of the present study was to investigate the interaction between ASA and BTZ in the MM1.S and RPMI-8226 myeloma cell lines, and clarify the underlying mechanisms through detecting the effects of ASA and BTZ on the Bcl-2, survivin and AKT proteins.

\section{Materials and methods}

Drugs and reagents. ASA (Sigma-Aldrich; Merck Millipore, Darmstadt, Germany) was dissolved in solution containing $10 \mathrm{~N}$ sodium hydroxide and adjusted to $\mathrm{pH}$ 7.0. BTZ (Selleck Chemicals, Houston, TX, USA) was dissolved in dimethyl sulfoxide at a final concentration of $50 \mu \mathrm{M}$.

All liquid culture media were acquired from Invitrogen; Thermo Fisher Scientific, Inc. (Waltham, MA, USA). The BCA protein assay kit was obtained from Beyotime Institute of Biotechnology (Nantong, China). Antibodies against GADPH and Bcl-2 were obtained from Goodhere Biotechnology Co., Ltd. (Hangzhou, China) and Sigma-Aldrich; Merck Millipore, respectively. Antibodies against Akt, p-Akt (Thr308) and p-Akt (Ser473) were obtained from CST Biological Reagents Company Limited (Shanghai, China). The secondary antibody was from EarthOx Company (San Francisco, CA USA). The EnoGene $^{\mathrm{TM}}$ total protein extraction kit was acquired from EnoGene (Nanjing, China). Phosphosafe ${ }^{\mathrm{TM}}$ extraction reagent was purchased from Merck Millipore. The chemiluminescent detection kit (Super-Signal West Femto substrate) was from Thermo Fisher Scientific, Inc.

Cell culture. The MM1.S cell line, harboring the K-Ras mutation, and RPMI-8226, harboring the N-Ras mutation, were selected, as the oncogenic mutations of the K- and N-Ras genes were found to exist in $50 \%$ of MM cases and correlated with aggressive disease, resistance to therapy and poor survival rates (27). The MM1.S human myeloma cell line was provided by Dr Lu-Gui Qiu (Hematology Hospital, Chinese Academy of Medical Science, Tianjin, China). The human MM cell line (RPMI-8226) was purchased from American Type Culture Collection (Manassas, VA, USA). The cells were cultured at $37^{\circ} \mathrm{C}$ in a water-saturated atmosphere of $95 \%$ air and $5 \% \mathrm{CO}_{2}$ in RPMI-1640 medium supplemented with $10 \%$ heat-inactivated FBS (Thermo Fisher Scientific, Inc.), $100 \mathrm{U} / \mathrm{ml}$ penicillin and $100 \mu \mathrm{g} / \mathrm{ml}$ streptomycin. When indicated, the cells were seeded at a confluence of $80 \%$ (serum-free medium was the vehicle) in 96-well or 6-well plates and treated with vehicle or ASA.

Cell proliferation assay. The cells were harvested and seeded into 96 -well plates $\left(1 \times 10^{4}\right.$ cells per well) in a total volume of $200 \mu \mathrm{l}$. According to the experimental design, the cells were divided into four groups, including untreated, ASA-treated, BTZ-treated and ASA+BTZ co-treated groups. The cells were then incubated for 24,48 and $72 \mathrm{~h}$ at $37^{\circ} \mathrm{C}$, respectively. Following treatment of the cells for indicated durations, Cell Counting Kit-8 (CCK-8) solution (Dojindo Molecular Technologies, Inc., Kumamoto, Japan) was added (20 $\mu 1$ per well) and the cells were incubated for $2 \mathrm{~h}$ at $37^{\circ} \mathrm{C}$. The plates were then read on an automated microplate spectrophotometer (DNM-9602; Perlong Medical Equipment Co., Ltd., Nanjing, China) at $450 \mathrm{~nm}$.

Cell apoptosis analysis. In the cell apotosis assay, Annexin-V-fluorescenin isothiocyanate (FITC; 1:250) and propidium iodide (PI; $1 \mu \mathrm{g} / \mathrm{ml}$ ) were used, according to the protocol of the Annexin V-FITC apoptosis assay kit (Beyotime Institute of Biotechnology). FITC specifically binds to the phosphatidyl serine residues on the cell membrane, whereas PI binds to DNA when the cell membrane becomes permeable. The cells were stained and analyzed using the FACScan system (BD Biosciences, Franklin Lakes, NJ, USA). The data were analyzed using CellQuest (BD Biosceinces) software. For each analysis, 10,000 events were recorded.

Drug interaction analysis. The ratio $(R)$ was used to analyze the interaction of the ASA and BTZ combination in the two cell lines, as previously described (28). The $R$ ratio was calculated as follows:

\section{$R=$ survival (ASA+BTZ) / (survival ASA x survival BTZ)}

If $R<0.8$, the combination was considered to be synergistic; if $0.8<R<1.2$, the combination was considered additive; if $R>1.2$, the combination was considered antagonistic.

Western blot analysis. The treated cells $\left(5-6 \times 10^{6}\right)$ were washed in PBS and then lysed in 2X Laemmli buffer. Protein concentrations were determined using the BCA assay kit.

Following boiling for $5 \mathrm{~min}$, equivalent quantities of protein (30-40 $\mu \mathrm{g}$ ) were separated on 8-12\% SDS-polyacrylamide gels and then transferred onto a nitrocellulose membrane $(0.45 \mu \mathrm{M})$. The membranes were blocked with $5 \%$ BSA in TBS/Tween $20(0.05 \% \mathrm{v} / \mathrm{v})$ for $1 \mathrm{~h}$, followed by incubation at $4^{\circ} \mathrm{C}$ overnight with primary antibodies against Bcl-2 (1:500 dilution), survivin (1:870 dilution), Akt (1:1,000 dilution), p-Akt (Thr308; 1:1,000 dilution) and p-Akt (Ser473; 1:1,000 
dilution). GADPH (1:1,000 dilution) served as a control. The membranes were washed three times with TBST and then incubated for $1 \mathrm{~h}$ at room temperature with horseradish peroxidase-conjugated anti-rabbit secondary antibodies $(1: 10,000$ dilution). The protein bands were visualized using enhanced chemiluminescence on a ChemiDoc ${ }^{\mathrm{TM}} \mathrm{XRS}^{+}$system with Image $\mathrm{Lab}^{\mathrm{TM}}$ software version 4.0 (Bio-Rad Laboratories, Inc., Hercules, CA, USA). The quantification of protein levels was performed by Gel-Pro Analyzer (version 4.0) and the integrated option density was used as the quantity for comparison.

Statistical analysis. All results in bar graphs are expressed as the mean + standard deviation obtained from at least three independent experiments. Statistical differences were evaluated using Student's $t$-test (paired) and one-way analysis of variance (ANOVA), as appropriate, using SPSS 13.0 (SPSS, Inc, Chicago, IL, USA). $\mathrm{P} \leq 0.05$ was considered to indicate a statistically significant difference. Differences determined to be significant using ANOVA were further analyzed using Turkey's pairwise comparison to detect specific differences between treatments.

\section{Results}

Co-treatment of ASA and BTZ reduces the proliferation of myeloma cells. ASA at a dose of $2.5 \mathrm{mM}$ was selected for use in experiments in the present study, as $2.5 \mathrm{mM}$ was the concentration measured in the serum of patients treated with ASA for chronic inflammatory diseases in a previous study (29). A BTZ concentration of $10 \mathrm{nM}$ was the plateau concentration following an initial peak at $\sim 300 \mathrm{nM}$ (for $1 \mathrm{~h}$ ) in the serum of BTZ-treated patients with MM in a previous study (30). Therefore, $10 \mathrm{nM}$ BTZ was used as the experimental concentration in the present study. The effects of ASA, BTZ and ASA in combination with BTZ on the proliferation of MM1.S and RPMI-8226 cells were evaluated using a CCK-8 assay.

As shown in Fig. 1A, the MM1.S cells treated with ASA+BTZ for 24, 48 and $72 \mathrm{~h}$ led to significant decreases $(\mathrm{P}<0.05)$ in cell proliferation $(78.3,85.1$ and $91.9 \%$, respectively), compared with the cells treated with ASA alone (52.7, 64.6 and $72.8 \%$, respectively) or BTZ alone (43.6, 64.1 and $75.1 \%$, respectively. However, no significant difference was found between the ASA-treated and BTZ-treated groups.

As shown in Fig. 1B, treatment with ASA+BTZ significantly $(\mathrm{P}<0.05)$ reduced the viability of the RPMI-8226 cells within 24,48 and $72 \mathrm{~h}$ (56.4, 73.9 and $88.5 \%$, respectively), compared with the cells exposed to ASA alone (43.4, 56.1 and $63.6 \%$, respectively) or BTZ alone (32.1, 42.9 and $68.4 \%$, respectively). Again, no statistical differences were found between the ASA-treated and BTZ-treated groups ( $\mathrm{P}>0.05)$.

The combination treatment of ASA and BTZ for 24, 48 and $72 \mathrm{~h}$ resulted in an additive effect on the inhibitory rate of MM1.S or RPMI-8226 cells, as $R$ was between 0.8 and 1.2 in the two cell lines, indicating that ASA enhanced the antimyleoma activity of BTZ.

Co-exposure of $A S A+B T Z$ augments the apoptotic rate of myelomacells. The present study used Annexin V-FITC/PI flow cytometry to determine whether the additive action of ASA and BTZ was correlated with the induction of cell apoptosis. The
A

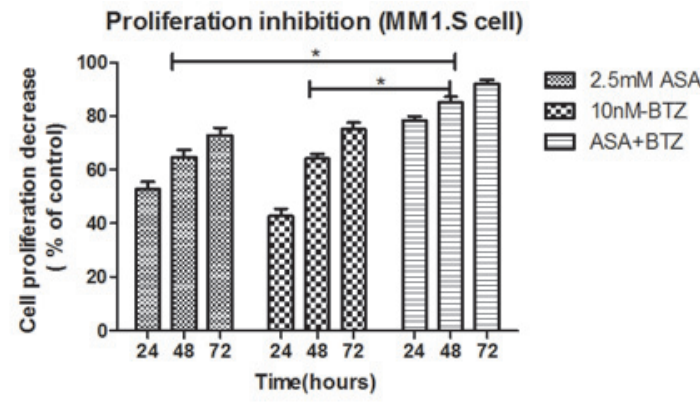

B

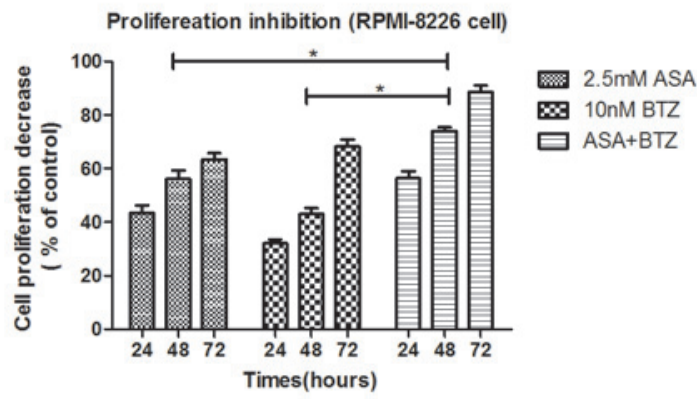

Figure 1. Cotreatment of ASA and BTZ reduces the proliferation of myeloma cells. (A) MM1.S cells and (B) RPMI-8226 cells were treated with ASA alone, BTZ alone and ASA+BTZ for 24, 48 and $72 \mathrm{~h}$, respectively. At each time point, the cell proliferation was determined using Cell-Counting Kit-8 analysis. Data are presented as the mean + standard deviation from independent experiments. ${ }^{*} \mathrm{P}<0.05$. ASA, aspirin; BTZ, bortezomib.

MM1.S and RPMI-8226 cells were incubated with ASA alone, BTZ alone, and with the combination of ASA and BTZ at $37^{\circ} \mathrm{C}$ for $48 \mathrm{~h}$. At the indicated duration, the cells were harvested for the Annexin V-FITC/PI assay using flow cytometry.

As shown in Fig. 2, the apoptotic rates of the untreated cells, and the cells treated with ASA alone, BTZ alone and with ASA and BTZ were 2.73, 44.8, 45.4 and 64.3\%, respectively. Co-exposure of the cells to ASA with BTZ led to higher rates of apoptosis, compared with the cells treated with either ASA or BTZ alone $(\mathrm{P}<0.05)$. No significant differences were found between the ASA-treated group and BTZ-treated group.

In the RPMI-8226 cells, the apoptotic rates were $3.43,36.1$, 57.9 and $77.2 \%$ in the untreated, ASA-treated, BTZ-treated and ASA+BTZ-treated cells, respectively. Similarly, co-treatment with ASA and BTZ triggered higher rates of cell apoptosis, compared with either ASA or BTZ alone $(\mathrm{P}<0.05)$. Unlike the MM1.S cells, BTZ treatment induced higher rates of apoptosis, compared with ASA treatment in the RPMI-8226 cells $(\mathrm{P}<0.05)$. Taken together, these findings revealed that co-exposure to ASA and BTZ augmented the apoptotic rate of myeloma cells, compared with ASA or BTZ treatment alone in myeloma cells.

ASA inhibits the phosphorylation of AKT induced by BTZ in myeloma cells. AKT is important in the carcinogenesis of MM via controlling cell survival and apoptosis (31). Aberrant AKT activation confers chemoresistance to dexamethasone (Dex) and BTZ in MM cells (24). In particular, the phosphorylation of Thr308 and Ser473 is essential in the activation of AKT. Therefore, the present study examined the effect of ASA and 
A
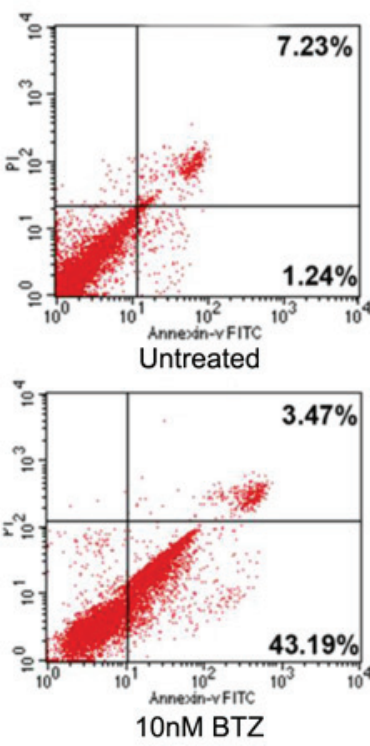

MM1.S cell
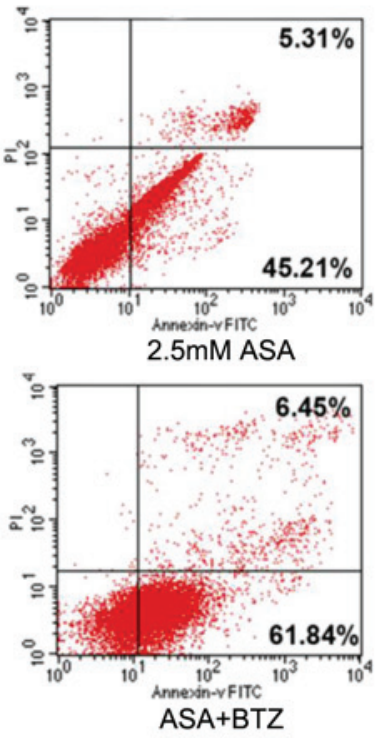
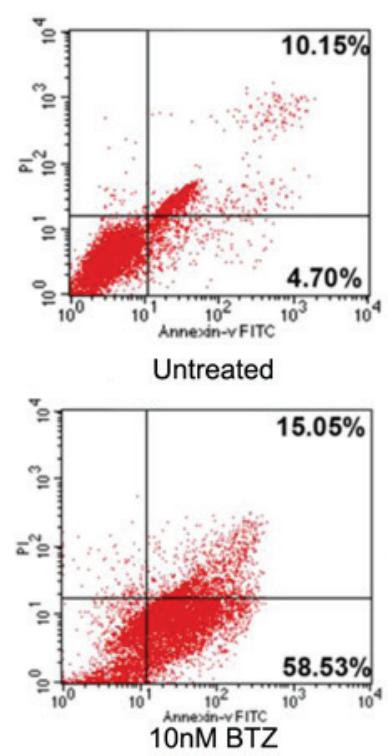

RPMI-8226 cell
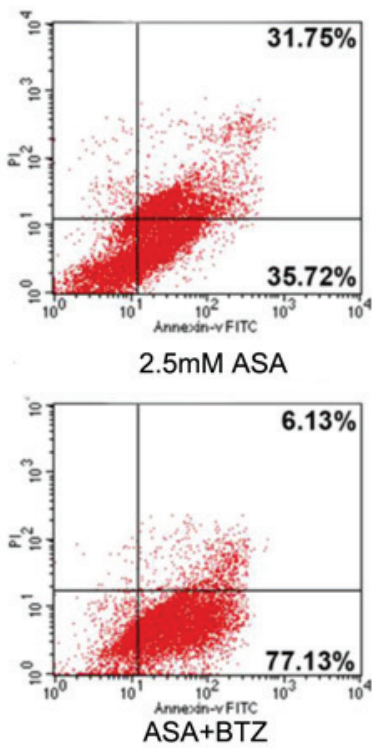

C

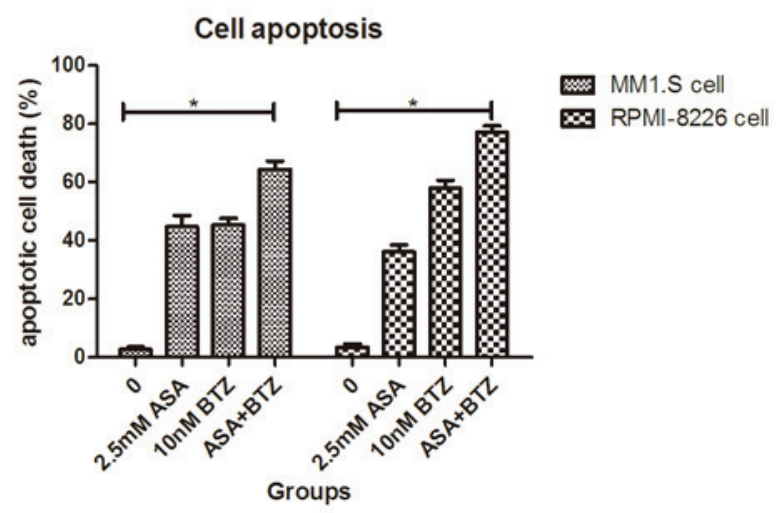

Figure 2. Effect of ASA in combination with BTZ on the apoptosis of MM1.S and RPMI-8226 cells. The two cell lines were treated with ASA alone, BTZ alone and ASA+BTZ for $48 \mathrm{~h}$. The cells were harvested to analyze apoptosis using flow cytometry. (A) Annexin V-FITC/PI assay of MM1.S cells. (B) Annexin V-FITC/PI assay of RPMI-8226 cells. Numbers in the respective area indicate the percentage of cells. (C) Apoptotic rates of MM1.S and RPMI-8226 cells induced by ASA alone, BTZ alone and ASA+BTZ. Results are expressed as the means + standard deviation from three independent experiments. $\mathrm{P}<0.05$. ASA, aspirin; BTZ, bortezomib; FITC; fluorescenin isothiocyanate; PI, propidium iodide.

BTZ alone or in combination on the phosphorylation of AKT in myeloma cells over $48 \mathrm{~h}$.

As shown in Fig. 3, the MM1.S and RPMI-8226 cells expressed high endogenous levels of $\mathrm{p}$-AKT, indicating the constitutive activation of AKT in the two cell lines. ASA treatment markedly decreased the levels of p-AKT, including Thr308 and Ser473, in the MM1.S and RPMI-8226 cells. Of note, exposure of the two cell lines to ASA almost completely inhibited the phosphorylation of AKT at Thr308. However, no significant changes were found in total AKT protein between the two cell lines. In addition, it was found that BTZ treatment led to the enhancement of p-AKT, including Thr308 and Ser473. Treatment with ASA in combination with BTZ markedly reduced the phosphorylation of AKT, particularly for Thr308, suggesting the potential activity of ASA in overcoming the resistance to BTZ in myeloma cells.

ASA downregulates the expression of survivin induced by BTZ. As a prominent anti-apoptotic molecule, increased levels of survivin contribute to uncontrolled cell proliferation and drug resistance in MM. Therefore; the present study investigated the effect of the ASA and BTZ combination on the level of survivin.

As shown in Fig. 4, exposure of the MM1.S and RPMI-8226 cells to ASA alone for $48 \mathrm{~h}$ led to significant decreases in survivin, compared with the untreated group. By contrast, BTZ treatment induced the upregulation of survivin in the MM1.S and RPMI-8226 cells. However, the combined treatment with ASA and BTZ decreased the protein levels of survivin in the MM1.S and RPMI-8226 cells, indicating that ASA may inhibit the upregulation of survivin triggered by BTZ.

ASA in combination with BTZ suppresses the expression of Bcl-2 in myeloma cells. As a classical anti-apoptotic gene, $\mathrm{Bcl}-2$ regulates apoptosis and cell death in MM, contributing to the pathogenesis, progression and chemoresistance of MM. Therefore, the present study examined the effect of treatment with ASA and/or BTZ on the protein expression of Bcl-2.

As shown in Fig. 5, incubation of the myeloma cells with ASA or BTZ for $48 \mathrm{~h}$ caused a marked downregulation in the level of $\mathrm{Bcl}-2$, compared with the corresponding vehicle. 
A

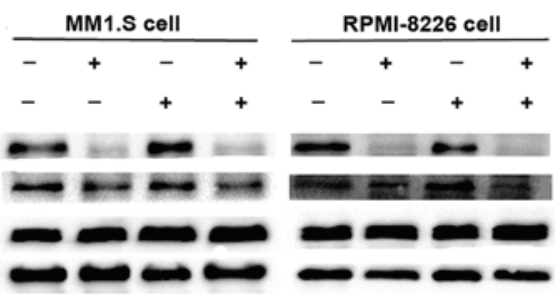

ASA (2.5mM)

BTZ (10.0nM)

p-AKT(Thr308)

p-AKT(Ser473)

AKT

GADPH

B

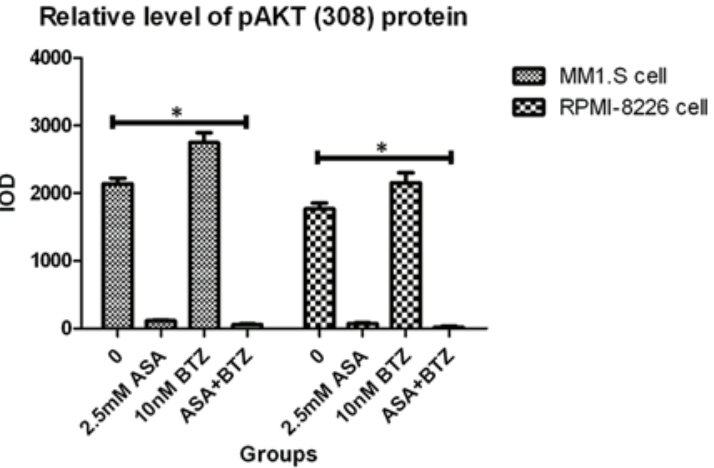

C

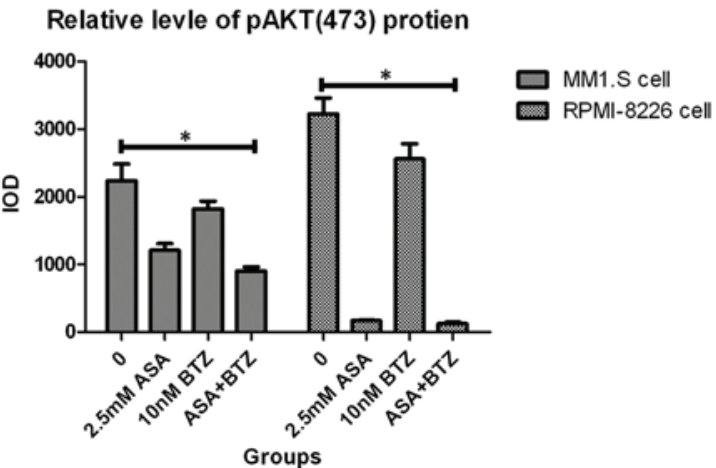

Figure 3. Effect of ASA and/or BTZ treatment on expression of p-AKT in myeloma cells. (A) MM1.S and RPMI-8226 cells were treated with ASA, BTZ and ASA+BTZ for $48 \mathrm{~h}$, respectively, following which the whole cell lysates were prepared using Phosphosafe ${ }^{\mathrm{TM}}$ extraction reagent. The levels of p-AKT (Thr 308 and Ser 473) and AKT were analyzed using western blot analysis with corresponding antibodies. Each blot is representative of three independent experiments. (B) Integrated optical density data of p-AKT(308) are presented as the mean \pm standard deviation. (C) Integrated optical density data of p-AKT(473) are presented as the mean \pm standard deviation. ${ }^{*} \mathrm{P}<0.05$ p-AKT, phosphorylated AKT; ASA, aspirin; BTZ, bortezomib; Bcl-2, B cell lyphoma-2.

Notably, exposure of myeloma cells to ASA+BTZ markedly decreased the protein level of Bcl-2, compared with ASA or BTZ treatment alone. These results supported the causal association between the suppression of $\mathrm{Bcl}-2$ protein and the apoptosis induced by ASA and/or BTZ in MM1.S and RPMI-8226 myeloma cells.

\section{Discussion}

At present, BTZ has been incorporated as leading agent into conditioning, consolidation and maintenance therapy for patients with MM (19). However, the prolonged administration of BTZ is associated with the development of drug resistance (20). The mechanisms underlying BTZ resistance in MM remain to be elucidated, however, they have been partly attributed to 'molecular side-effects', including the induction
A

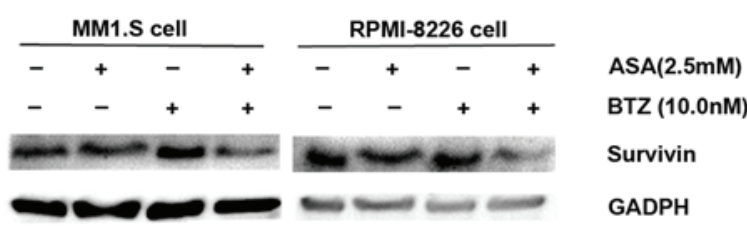

B Relative Level of Survivin Protein

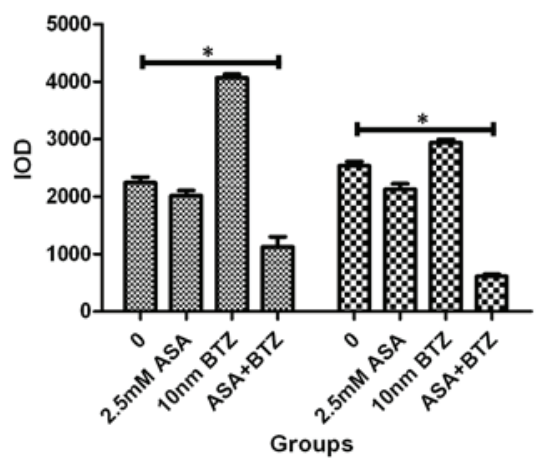

MM1.S cell $\infty$ RPMI-8226 cell

Figure 4. Expression of Survivin in myeloma cells treated with ASA and/or BTZ. (A) The MM1.S and RMPI-8226 cells were incubated with ASA, BTZ and ASA+BTZ for $48 \mathrm{~h}$, respectively. The protein expression of Survivin was determined using western blot analysis. Each blot is representative of three independent experiments. (B) Integrated optical density data are presented as the mean \pm standard deviation. ${ }^{*} \mathrm{P}<0.05$. ASA, aspirin; BTZ, bortezomib.

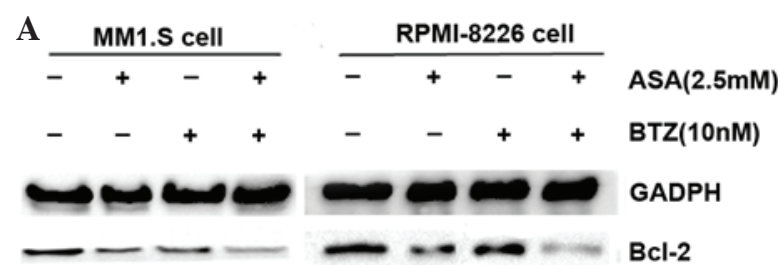

B

Relative level of $\mathrm{Bcl}-2$ Protein

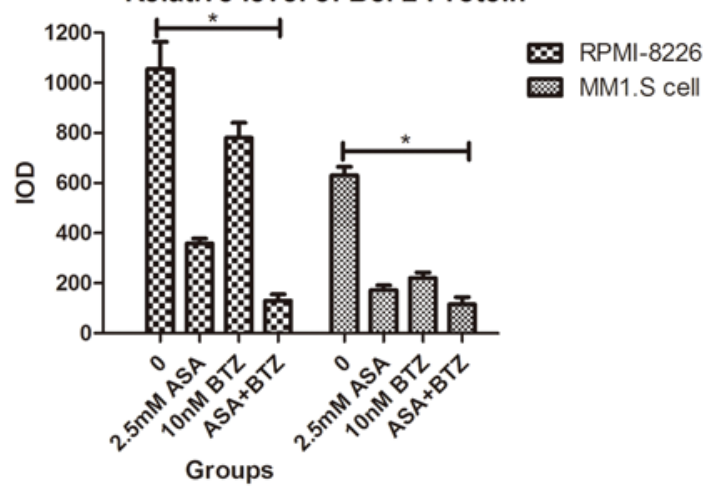

Figure 5. Effect of ASA and/or BTZ on the expression of Bcl-2 in myeloma cells. (A) The MM1.S and RPMI-8226 cells were exposed to ASA, BTZ and ASA+BTZ for $48 \mathrm{~h}$, respectively. The protein level of Bcl-2 was analyzed using western blot analysis. Each blot is representative of three independent experiments. (B) Integrated optical density data are presented as the mean \pm standard deviation. ${ }^{*} \mathrm{P}<0.05$. ASA, aspirin; BTZ, bortezomib; Bcl-2, B cell lymphoma- 2 .

of AKT activation and upregulation of survivin (24), which attenuate the cytotoxic action of BZT against MM cells and can lead to chemoresistance. Therefore, identifying how to enhance the antimyeloma activity of BTZ, particularly in patients with relapsed or refractory MM, is required. The 
antineoplastic role of ASA has been verified in a wide range of solid tumor types (32-36). Our previous study reported that ASA exerted antimyeloma activity in vitro and in vivo accompanying the thromboprophylactic profile (18). In the present study, the chemosensitive effect of ASA was examined in myeloma cells treated with BTZ based on the 'molecular side-effects'.

The concentrations of $2.5 \mathrm{mM}$ ASA and $10 \mathrm{nM}$ BTZ were selected as the experimental concentrations in the present study, as they resemble the serum concentrations in patients with MM with important clinical implications $(29,30)$. Initially, the present study found that ASA in combination with BTZ resulted in enhanced inhibition of cell proliferation, compared with ASA or BTZ alone in MM1.S and RPMI-8226 cells, and occurred in a time-dependent manner. Subsequent interaction analysis revealed an additive effect, indicating that ASA may potentiate the antimyeloma property of BTZ. In accordance, the co-administration of ASA and BTZ triggered higher apoptotic rates, compared with treatment with ASA or BTZ alone in the two cell lines. These results supported the hypothesis that ASA has a chemosensitive effect in BTZ-treated myeloma cells.

As a serine-threonine kinase, AKT is a key molecule of the phosphoinositide 3-kinase/AKT signaling pathway. Once recruited to the plasma membrane, AKT is activated by phosphorylation at the Ser473 and Thr308 residues, and then regulates the function of several cellular proteins involved in survival/apoptosis, differentiation and proliferation, including mammalian target of rapamycin, $N F-\kappa B$ and glycogen synthase kinase-3 (37). Several studies have provided evidence that the aberrant activation of AKT is important in the pathogenesis of various types of cancer, including MM (38-41). The ectopic activation of AKT has been documented in $~ 50 \%$ patients with MM. In addition, p-AKT renders anti-apoptotic activity to $\mathrm{MM}$ cells, indicating the importance of AKT activation in the carcinogenesis of MM (42). p-AKT is also associated with the prognosis in MM (38). These findings provide compelling evidence that AKT may be a potential therapeutic target in MM treatment. In the present study, treatment with ASA alone almost completely inhibited the phosphorylation of AKT (Thr308) and partly suppressed the phosphorylation of AKT (Ser473) in MM1.S and RPMI-8226 cells, indicating that AKT was the anticancer target of ASA in myeloma cells. By contrast, exposure of the myeloma cells to BTZ alone induced the phosphorylation of AKT, which was in agreement with a report by Hideshima et al (24). Of note, treatment with ASA combined with BTZ markedly inhibited the levels of p-AKT (Thr308 and Ser473), particularly for Thr308. These data suggested that ASA may augment the cytotoxic activity of BTZ against myeloma cells, and may even overcome the chemoresistance of BTZ in refractory/relapsed MM.

Survivin is also involved in inhibiting apoptosis, promoting proliferation and enhancing invasion in malignancies. Survivin has been reported to be overexpressed ubiquitously in cells in several types of cancer, including breast cancer (43), lung cancer (44) and MM (45). Furthermore, elevated levels of survivin have been correlated with poor prognosis, decreased survival and chemotherapy resistance in MM cells $(45,46)$. Accordingly, the inhibition of survivin may be an efficient form of therapy in patients with MM. In the present study, it was demonstrated that BTZ alone was capable of increasing the level of survivin in MM1.S and RPMI-8226 cells, which was in accordance with the results reported in human non-small cell lung cancer cells by Liu et al (47). By contrast, the concurrent use of ASA and BTZ substantially inhibited the expression of survivin in the two cell lines. These findings provided convincing evidence that ASA may increase the cytotoxicity of BTZ to myeloma cells, in part, via the inhibition of survivin.

Cell apoptosis is correlated with a broad spectrum of cellular factors and pathophysiologic pathways. The Bcl-2 family is the most prominent in the process of apoptosis. In addition, Bcl-2, as a classical anti-apoptotic protein, has been confirmed to be overexpressed in a diverse range of types of cancer in humans (48-50). The Bcl-2 protein is expressed at high levels in patients with myeloma and is implicated in the regulation of chemosensitivity. Dex has been used for the treatment of MM for $>50$ years. Dex has also been incorporated into agents in combination with BTZ, thalidomide or lenalidomide. However, elevated expression of Bcl-2 confers chemoresistance to Dex in myeloma cells $(51,52)$. In the present study, ASA exhibited antineoplastic activity against Dex-sensitive MM1.S cells and Dex-resistant RPMI-8226 cells. The western blot assay revealed that treatment with either ASA or BTZ alone downregulated the level of Bcl-2. Furthermore, ASA in combination with BTZ led to a marked decrease in the protein level of Bcl-2, compared with either ASA or BTZ alone, suggesting that ASA in combination with BTZ may be a promising treatment regimen for patients with $\mathrm{MM}$ with elevated expression of $\mathrm{Bcl}-2$. Thus, the rationale of a combined regimen of Dex and BTZ in treating MM may be, in part, based on the ability of BTZ to inhibit Bcl-2. These findings indicated that BTZ may abrogate chemoresistance to Dex mediated by enhanced Bcl-2, which was consistent with reports by Ailawadhi et al (53) and Pei et al (54).

Perifosine (PERI), a novel synthetic alkylphospholipid, targets Akt and exerts potent antineoplastic activity in a wide variety of neoplasms, particularly MM and colorectal carcinoma (55). PERI also induces the significant downregulation of survivin in human myeloma cells (56). Clinical observations have found that a treatment regimen of PERI in combination with BTZ showed marked activity, with an overall response rate (ORR) of 32 and $65 \%$, and median overall survival (mOS) of 22.5 and 30.4 months in BTZ-refractory and BTZ-relapsed patients, respectively (57). Encouraged by this data, a placebo-controlled phase III study was designed to evaluate the effect of adding PERI (50 mg daily) to BTZ and DEX in patients with MM who had relapsed following a BTZ-based regimen and who had received between one and four previous treatment regimens. However, this phase III experiment showed no benefit in PFS or ORR when PERI was added to BTZ and DEX in patients with resistant, relapsed and refractory MM. Furthermore, the mOS for PERI was 141.9 weeks and for the placebo was 83.3 weeks, which showed favorable results for PERI, but without statistical significance $(\mathrm{P}=0.356)(58)$, therefore, the investigation was discontinued.

Coincidently, in the present study, it was found that ASA also inhibited the expression of survivin and the phosphorylation of AKT induced by BTZ in myeloma cells, which explained the enhanced cytotoxicity of ASA+BTZ against 
myeloma cells, compared with either agent alone. Notably, ASA retained its thromboprophylactic property, which was deficient in the case with PERI; treatment with PERI $(150 \mathrm{mg}$ daily) caused increased creatinine in $30-60 \%$ of patients with MM (59), whereas the continued administration of ASA may have a protective effect against renal injury (60). Thus, ASA may be a more superior candidate to augment the antimyeloma activity of BTZ, compared with PERI.

In conclusion, the present study demonstrated that the concurrent administration of ASA and BTZ caused higher levels of proliferative inhibition and apoptotic rates, compared with either agent alone in myeloma cells in vitro, which indicated the chemosensitivity towards ASA in MM treated by BTZ. The underlying mechanisms included the suppression of survivin and AKT phosphorylation by ASA induced by BTZ, together with the inhibition of $\mathrm{Bcl}-2$, suggesting the potential of a regimen comprising ASA+BTZ-containing chemotherapy in patients with refractory or relapsed MM.

\section{Acknowledgements}

This study was funded by the National Nature Science Foundation of China (grant no. 81460037) and the Medical and Healthy Research Foundation of Nanjing Military Area of China (grant no. 14ZD31).

\section{References}

1. Siegel R, Ma J, Zou Z and Jemal A: Cancer statistics, 2014. CA Cancer J Clin 64: 9-29, 2014.

2. Chen W, Zheng R, Baade PD, Zhang S, Zeng H, Bray F, Jemal A, $\mathrm{Yu}$ XQ and He J: Cancer statistics in China, 2015. CA Cancer J Clin 66: 115-132, 2016.

3. Sonneveld P and Broijl A: Treatment of relapsed and refractory multiple myeloma. Haematologica 101: 995, 2016.

4. Morgan GJ and Davies FE: Role of thalidomide in the treatment of patients with multiple myeloma. Crit Rev Oncol Hematol 1: (88 Supp 1) S14-S22, 2013.

5. Bagratuni T, Kastritis E, Politou M, Roussou M, Kostouros E, Gavriatopoulou M, Eleutherakis-Papaiakovou E, Kanelias N, Terpos E and Dimopoulos MA: Clinical and genetic factors associated with venous thromboembolism in myeloma patients treated with lenalidomide-based regimens. Am J Hematol 88: 765-770, 2013.

6. Palumbo A, Cavo M, Bringhen S, Zamagni E, Romano A Patriarca F, Rossi D, Gentilini F, Crippa C, Galli M, et al: Aspirin, warfarin, or enoxaparin thromboprophylaxis in patients with multiple myeloma treated with thalidomide: A phase III, open-label, randomized trial. J Clin Oncol 29: 986-993, 2011

7. Niesvizky R, Martínez-Baños D, Jalbrzikowski J, Christos P, Furst J, De Sancho M, Mark T, Pearse R, Mazumdar M, Zafar F, et al: Prophylactic low-dose aspirin is effective antithrombotic therapy for combination treatments of thalidomide or lenalidomide in myeloma. Leuk Lymphoma 48: 2330-2337, 2007.

8. Araujo JL, Altorki NK, Sonett JR, Rodriguez A, Sungur-Stasik K, Spinelli CF, Neugut AI and Abrams JA: Prediagnosis aspirin use and outcomes in a prospective cohort of esophageal cancer patients. Therap Adv Gastroenterol 9: 806-814.

9. Murphy C, Turner N, Wong HL, Sinnathamby M, Tie J, Lee B, Desai J, Skinner I, Christie M and Hutchinson R: Examining the impact of regular aspirin use and PIK3CA mutations on survival in stage 2 colon cancer. Intern Med J: Nov 1, 2016 (Epub ahead of print).

10. Hochmuth F, Jochem M and Schlattmann P: Meta-analysis of aspirin use and risk of lung cancer shows notable results. Eur J Cancer Prev 25: 259-268, 2016.

11. Bradley MC, Black A, Freedman AN and Barron TI: Prediagnostic aspirin use and mortality in women with stage I to III breast cancer: A cohort study in the Prostate, Lung, Colorectal, and Ovarian Cancer Screening Trial. Cancer 122: 2067-2075, 2016.
12. Kim YI, Kim SY, Kim JH, Lee JH, Kim YW, Ryu KW, Park $\mathrm{JH}$ and Choi IJ: Long-term low-dose aspirin use reduces gastric cancer incidence: A nationwide cohort study. Cancer Res Treat 48: 798-805, 2016.

13. Xiang S, Sun Z, He Q, Yan F, Wang Y and Zhang J: Aspirin inhibits ErbB2 to induce apoptosis in cervical cancer cells. Med Oncol 27: 379-387, 2010.

14. Park IS, Jo JR, Hong H, Nam KY, Kim JB, Hwang SH, Choi MS, Ryu NH, Jang HJ, Lee SH, et al: Aspirin induces apoptosis in YD-8 human oral squamous carcinoma cells through activation of caspases, down-regulation of Mcl-1, and inactivation of ERK-1/2 and AKT. Toxicol In Vitro 24: 713-720, 2010.

15. Ararat E, Sahin I and Altundag K: Mechanisms behind the aspirin use and decreased breast cancer incidence. J BUON 16: 180,2011

16. Im SR and Jang YJ: Aspirin enhances TRAIL-induced apoptosis via regulation of ERK1/2 activation in human cervical cancer cells. Biochem Biophys Res Commun 424: 65-70, 2012.

17. Lim WY, Chuah KL, Eng P, Leong SS, Lim E, Lim TK, $\mathrm{Ng}$ A, Poh WT, Tee A, Teh M, et al: Aspirin and non-aspirin non-steroidal anti-inflammatory drug use and risk of lung cancer. Lung Cancer 77: 246-251, 2012.

18. Ding JH, Yuan LY, Huang RB and Chen GA: Aspirin inhibits proliferation and induces apoptosis of multiple myeloma cells through regulation of $\mathrm{Bcl}-2$ and Bax and suppression of VEGF. Eur J Haematol 93: 329-339, 2014.

19. Chen D, Frezza M, Schmitt S, Kanwar J and Dou QP: Bortezomib as the first proteasome inhibitor anticancer drug: Current status and future perspectives. Curr Cancer Drug Targets 11: 239-253, 2011.

20. Chauhan D, Tian Z, Nicholson B, Kumar KG, Zhou B, Carrasco R, McDermott JL, Leach CA, Fulcinniti M, Kodrasov MP, et al: A small molecule inhibitor of ubiquitin-specific protease-7 induces apoptosis in multiple myeloma cells and overcomes bortezomib resistance. Cancer Cell 22: 345-358, 2012.

21. Oerlemans R, Franke NE, Assaraf YG, Cloos J, van Zantwijk I, Berkers CR, Scheffer GL, Debipersad K, Vojtekova K, Lemos C, et al: Molecular basis of bortezomib resistance: Proteasome subunit beta5 (PSMB5) gene mutation and overexpression of PSMB5 protein. Blood 112: 2489-2499, 2008.

22. Kuhn DJ, Berkova Z, Jones RJ, Woessner R, Bjorklund CC, Ma W, Davis RE, Lin P, Wang H, Madden TL, et al: Targeting the insulin-like growth factor-1 receptor to overcome bortezomib resistance in preclinical models of multiple myeloma. Blood 120: 3260-3270, 2012.

23. Gu JL, Li J, Zhou ZH, Liu JR, Huang BH, Zheng D and Su C: Differentiation induction enhances bortezomib efficacy and overcomes drug resistance in multiple myeloma. Biochem Biophys Res Commun 420: 644-650, 2012.

24. Hideshima T, Catley L, Yasui H, Ishitsuka K, Raje N, Mitsiades C, Podar K, Munshi NC, Chauhan D, Richardson PG and Anderson KC: Perifosine, an oral bioactive novel alkylphospholipid, inhibits Akt and induces in vitro and in vivo cytotoxicity in human multiple myeloma cells. Blood 107: 4053-4062, 2006.

25. Uddin S, Ahmed M, Hussain A, Assad L, Al-Dayel F, Bavi P, Al-Kuraya KS and Munkarah A: Cyclooxygenase-2 inhibition inhibits PI3K/AKT kinase activity in epithelial ovarian cancer. Int J Cancer 126: 382-394, 2010.

26. Yang L, Zhu H, Liu D, Liang S, Xu H, Chen J, Wang X and Xu Z: Aspirin suppresses growth of human gastric carcinoma cell by inhibiting survivin expression. J Biomed Res 25: 246-253, 2011.

27. Chng WJ, Gonzalez-Paz N, Price-Troska T, Jacobus S, Rajkumar SV, Oken MM, Kyle RA, Henderson KJ, Van Wier S, Greipp P, et al: Clinical and biological significance of RAS mutations in multiple myeloma. Leukemia 22: 2280-2284, 2008.

28. Fischel JL, Formento P and Milano G: Epidermal growth factor receptor double targeting by a tyrosine kinase inhibitor (Iressa) and a monoclonal antibody (Cetuximab). Impact on cell growth and molecular factors. Br J Cancer 92: 1063-1068, 2005.

29. Book Review: Goodman and Gilman's the Pharmacological Basis of Therapeutics: Digital Edition, 11th Edition. 40: 1218, 2006.

30. Terpos E, Roussou M and Dimopoulos MA: Bortezomib in multiple myeloma. Expert Opin Drug Metab Toxicol 4: 639-654, 2008.

31. Harvey RD and Lonial S: PI3 kinase/AKT pathway as a therapeutic target in multiple myeloma. Future Oncol 3: 639-647, 2007.

32. Choe KS and Liauw SL: Effects of aspirin on cancer initiation and progression. Expert Rev Anticancer Ther 13: 115-117, 2013.

33. Wilson JC, Murray LJ, Hughes CM, Black A and Anderson LA: Non-steroidal anti-inflammatory drug and aspirin use and the risk of head and neck cancer. Br J Cancer 108: 1178-1181, 2013. 
34. Rothwell PM: Alternate-day, low-dose aspirin and cancer risk. Ann Intern Med 159: 148-150, 2013.

35. Jonsson F, Yin L, Lundholm C, Smedby KE, Czene K and Pawitan Y: Low-dose aspirin use and cancer characteristics: A population-based cohort study. Br J Cancer 109: 1921-1925, 2013

36. Algra AM and Nortier JW: Aspirin and cancer: Evidence of a prophylactic effect. Ned Tijdschr Geneeskd 157: A5189, 2013 (In Dutch).

37. Coutte L, Dreyer C, Sablin MP, Faivre S and Raymond E: PI3K-AKT-mTOR pathway and cancer. Bull Cancer 99: 173-180, 2012 (In French).

38. Hsu J, Shi Y, Krajewski S, Renner S, Fisher M, Reed JC, Franke TF and Lichtenstein A: The AKT kinase is activated in multiple myeloma tumor cells. Blood 98: 2853-2855, 2001.

39. Hasson SP, Rubinek T, Ryvo L and Wolf I: Endocrine resistance in breast cancer: Focus on the phosphatidylinositol 3 -Kinase/Akt/mammalian target of rapamycin signaling pathway. Breast Care (Basel) 8: 248-255, 2013.

40. Bi LK, Zhou N, Liu C, Lu FD, Lin TX, Xuan XJ, Jiang C, Han JL, Huang H, Zhang CX, et al: Kidney cancer cells secrete IL-8 to activate Akt and promote migration of mesenchymal stem cells. Urol Oncol 32: 607-612, 2014.

41. Ito S, Igishi T, Takata M, Ueda Y, Matsumoto S, Kodani M, Takeda K, Izumi H, Sakamoto T, Yamaguchi K, et al: Synergistic cell growth inhibition by the combination of amrubicin and Akt-suppressing agents in K-ras mutation-harboring lung adenocarcinoma cells: Implication of EGFR tyrosine kinase inhibitors. Int J Oncol 44: 685-692, 2014

42. Steinbrunn T, Stühmer T, Gattenlöhner S, Rosenwald A Mottok A, Unzicker C, Einsele H, Chatterjee M and Bargou RC: Mutated RAS and constitutively activated Akt delineate distinct oncogenic pathways, which independently contribute to multiple myeloma cell survival. Blood 117: 1998-2004, 2011.

43. Xu C, Yamamoto-Ibusuki M, Yamamoto Y, Yamamoto S, Fujiwara S, Murakami K, Okumura Y, Yamaguchi L, Fujiki Y and Iwase $\mathrm{H}$ : High survivin mRNA expression is a predictor of poor prognosis in breast cancer: A comparative study at the mRNA and protein level. Breast Cancer 21: 482-490, 2014.

44. Wang M, Liu BG, Yang ZY, Hong X and Chen GY: Significance of survivin expression: Prognostic value and survival in stage III non-small cell lung cancer. Exp Ther Med 3: 983-988, 2012.

45. Tsubaki M, Satou T, Itoh T, Imano M, Komai M, Nishinobo M, Yamashita M, Yanae M, Yamazoe Y and Nishida S: Overexpression of MDR1 and survivin, and decreased Bim expression mediate multidrug-resistance in multiple myeloma cells. Leuk Res 36: 1315-1322, 2012.

46. Tsubaki M, Komai M, Itoh T, Imano M, Sakamoto K, Shimaoka H, Takeda T, Ogawa N, Mashimo K, Fujiwara D, et al: By inhibiting Src, verapamil and dasatinib overcome multidrug resistance via increased expression of Bim and decreased expressions of MDR1 and survivin in human multidrug-resistant myeloma cells. Leuk Res 38: 121-130, 2014

47. Liu X, Yue P, Chen S, Hu L, Lonial S, Khuri FR and Sun SY: The proteasome inhibitor PS-341 (bortezomib) up-regulates DR5 expression leading to induction of apoptosis and enhancement of TRAIL-induced apoptosis despite up-regulation of c-FLIP and survivin expression in human NSCLC cells. Cancer Res 67: 4981-4988, 2007.
48. Geng M, Wang L and Li P: Correlation between chemosensitivity to anticancer drugs and Bcl-2 expression in gastric cancer. Int J Clin Exp Pathol 6: 2554-2559, 2013.

49. Du P, Cao H, Wu HR, Zhu BS, Wang HW, Gu CW, Xing CG and Chen W: Blocking Bcl-2 leads to autophagy activation and cell death of the HEPG2 liver cancer cell line. Asian Pac J Cancer Prev 14: 5849-5854, 2013.

50. Yang D, Chen MB, Wang LQ, Yang L, Liu CY and Lu PH: Bcl-2 expression predicts sensitivity to chemotherapy in breast cancer: A systematic review and meta-analysis. J Exp Clin Cancer Res 32: 105, 2013.

51. Iyer R, Ding L, Batchu RB, Naugler S, Shammas MA and Munshi NC: Antisense p53 transduction leads to overexpression of bcl-2 and dexamethasone resistance in multiple myeloma. Leuk Res 27: 73-78, 2003.

52. Gazitt Y, Fey V, Thomas C and Alvarez R: Bcl-2 overexpression is associated with resistance to dexamethasone, but not melphalan, in multiple myeloma cells. Int J Oncol 13: 397-405, 1998.

53. Ailawadhi S, Miecznikowski J, Gaile DP, Wang D, Sher T, Mulligan G, Bryant B, Wilding GE, Mashtare T, Stein L, et al: Bortezomib mitigates adverse prognosis conferred by Bcl-2 overexpression in patients with relapsed/refractory multiple myeloma. Leuk Lymphoma 53: 1174-1182, 2012.

54. Pei XY, Dai Y and Grant S: The proteasome inhibitor bortezomib promotes mitochondrial injury and apoptosis induced by the small molecule Bcl-2 inhibitor HA14-1 in multiple myeloma cells. Leukemia 17: 2036-2045, 2003.

55. Vink SR, Schellens JH, van Blitterswijk WJ and Verheij M: Tumor and normal tissue pharmacokinetics of perifosine, an oral anti-cancer alkylphospholipid. Invest New Drugs 23: 279-286, 2005.

56. Hideshima T, Catley L, Raje N, Chauhan D, Podar K, Mitsiades C, Tai YT, Vallet S, Kiziltepe T, Ocio E, et al: Inhibition of Akt induces significant downregulation of survivin and cytotoxicity in human multiple myeloma cells. Br J Haematol 138: 783-791, 2007.

57. Richardson PG, Wolf J, Jakubowiak A, Zonder J, Lonial S, Irwin D, Densmore J, Krishnan A, Raje N, Bar M, et al: Perifosine plus bortezomib and dexamethasone in patients with relapsed/refractory multiple myeloma previously treated with bortezomib: Results of a multicenter phase I/II trial. J Clin Oncol 29: 4243-4249, 2011

58. Richardson PG, Nagler A, Dina BY and Ashraf Badros: Randomized placebo-controlled phase III study of perifosine combined with bortezomib and dexamethasone in relapsed, refractory multiple myeloma patients previously treated with bortezomib. ASH Annual Meeting abstract 3189, 2013.

59. Jakubowiak AJ, Richardson PG, Zimmerman T, Alsina M, Kaufman JL, Kandarpa M, Kraftson S, Ross CW, Harvey C, Hideshima T, et al: Perifosine plus lenalidomide and dexamethasone in relapsed and relapsed/refractory multiple myeloma: A phase I multiple myeloma research consortium study. $\mathrm{Br}$ J Haematol 158: 472-480, 2012.

60. Gerrah R, Ehrlich S, Tshori S and Sahar G: Beneficial effect of aspirin on renal function in patients with renal insufficiency postcardiac surgery. J Cardiovasc Surg (Torino) 45: 545-550, 2004. 